\title{
Fair ball-in the park
}

T his reviewers' reading of the scenario assumes: (1) the "Research Committee of the College of Agriculture" (COA) is not a separate IACUC committee, and (2) that exemption of IACUC oversight of research "on and for the benefit of agricultural animals" maintained by the Schools of Medicine and Veterinary Medicine also reflects the position of the COA. If so, then the rational for exclusion of oversight is likely based upon the definitions in the Animal Welfare Act that "Federal facilities, elementary and secondary schools, and agricultural research institutions" are exempt, and that nanobody production is a use to "improve animal nutrition, breeding, management, or production efficiency, or for improving the quality of food or fiber."

Did the schools respond appropriately? No. The Guide for the Care and Use of Agricultural Animals in Research and Teaching $(\mathrm{Ag} \text { Guide })^{1}$ requires an IACUC; presumably, the COA's Research Committee is not such a committee. As a research university receiving public funding, adherence to the principals of the Ag Guide should be the minimal benchmarks. These are enumerated in its first paragraph: "Because a variety of management systems and physical accommodations may be used for agricultural animals, an understanding of the husbandry needs of each species and of the particular requirements of agricultural research and teaching is essential for an effective institutional program of agricultural animal care and use. Critical components of such a program should include: 1) clearly established lines of authority and responsibility; 2) an active Institutional Animal Care and Use Committee (IACUC); 3) procedures for self monitoring of the IACUC through semi-annual review of programs and facility oversight by the institutional officer; 4 ) appropriately maintained facilities for proper management, housing, and support of animals; 5) an adequate program of veterinary care; and 6) training and occupational health programs for individuals who work with the animals."

Is this biomedical or agricultural research? Llamas are defined by the USDA as "farm animals" when used solely for work or pack purposes. Antibody production (nanobody) is neither food nor fiber research but could be interpreted as being used "for improving animal nutrition, breeding, management, or production efficiency, or for improving the quality of food or fiber" as described for Dr. Zymansky's research scope. Absent more specific details of the research, I am conflicted to classify this as agricultural despite BIV being a disease of agricultural animals. I suspect the USDA would consider this to be nanobody production, not consider the ultimate use of the nanobodies, and classify the research as biomedical. Depending on the antigens and adjuvants used the final disposition of these animals may also be regulatory restricted (i.e. exclusion from human food chain) with such oversight best assured by an IACUC supported by other institutional expertise such as a Biosafety Officer and Attending Veterinarian.

Did the site visitors overstep their authority? No. The cause of the skin condition was not known or documented and as such posed a potential threat to the other animals in the barn. The lack of awareness by the barn manager is a possible indicator of inadequate daily health assessment, caretaker training, or veterinary oversight. That the veterinarians contacted Dr. Zymansky does not ensure adequate veterinary care. The clinical veterinarian and attending veterinarian should have been contacted. Even if the llamas are "agricultural," there is a potential impact on biomedical animals and thus IACUC oversight is appropriate. Oversight of all animals must be provided regardless of whether they are biomedical or agricultural.

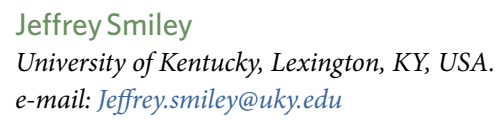

Published online: 22 January 2019 https://doi.org/10.1038/s41684-018-0224-3

\footnotetext{
References

1. Federation of Animal Science Societies (FASS). Guide for the Care and Use of Agricultural Animals in Research and Teaching. 3rd edn. (Federation of Animal Science Societies, Champaign, IL, 2010).
} 